\title{
Spatial and Temporal Organization of Ensemble Representations for Different Odor Classes in the Moth Antennal Lobe
}

\author{
Hong Lei, Thomas A. Christensen, and John G. Hildebrand \\ Arizona Research Laboratories, Division of Neurobiology, University of Arizona, Tucson, Arizona 85721
}

In the insect antennal lobe, odor discrimination depends on the ability of the brain to read neural activity patterns across arrays of uniquely identifiable olfactory glomeruli. Less is understood about the complex temporal dynamics and interglomerular interactions that underlie these spatial patterns. Using neural-ensemble recording, we show that the evoked firing patterns within and between groups of glomeruli are odor dependent and organized in both space and time. Simultaneous recordings from up to 15 units per ensemble were obtained from four zones of glomerular neuropil in response to four classes of odorants: pheromones, monoterpenoids, aromatics, and aliphatics. Each odor class evoked a different pattern of excitation and inhibition across recording zones. The excitatory response field for each class was spatially defined, but inhibitory activity was spread across the antennal lobe, reflecting a center-surround organization. Some chemically related odorants were not easily distinguished by their spatial patterns, but each odorant evoked transient synchronous firing across a uniquely different subset of ensemble units. Examination of 535 cell pairs revealed a strong relationship between their recording positions, temporal correlations, and similarity of odor response profiles. These findings provide the first definitive support for a nested architecture in the insect olfactory system that uses both spatial and temporal coordination of firing to encode chemosensory signals. The spatial extent of the representation is defined by a stereotyped focus of glomerular activity for each odorant class, whereas the transient temporal correlations embedded within the ensemble provide a second coding dimension that can facilitate discrimination between chemically similar volatiles.

Key words: odor discrimination; ensemble recording; glomerulus; olfaction; spatiotemporal coding; synchrony

\section{Introduction}

The spatial and temporal patterns of neural activity triggered by different scents at the initial stages of olfactory processing in the brain have been the subject of intense investigation in diverse animal models for well over half a century (Adrian, 1942). In an ongoing effort to decipher the neural coding strategies underlying the central processing of olfactory information, an increasing number of studies in the vertebrate olfactory bulb $(\mathrm{OB})$ and insect antennal lobe (AL) rely on imaging methods that reveal odor-dependent patterns of activity across olfactory glomeruli. Odorants possessing different functional groups or hydrocarbon-chain features evoke unique but overlapping activity patterns, whereas the patterns for odorants with similar chemical structure are less distinguishable (Rubin and Katz, 1999; Uchida et al., 2000; Linster et al., 2001; Wachowiak and Cohen, 2001; Johnson et al., 2002; Sachse and Galizia, 2002; Meijerink et al., 2003). How, then, does the brain discriminate structurally similar

Received Sept. 6, 2004; revised Oct. 21, 2004; accepted 0ct. 30, 2004.

This work was supported by National Institute on Deafness and Other Communication Disorders Grants DC-05652 (T.A.C.) and DC-02751 (J.G.H.). We thank the many talented individuals in our laboratory who have contributed to this work, especially Andrew Dacks, Suzanne Mackzum, Vince Pawlowski, and Heather Stein.

Correspondence should be addressed to Dr. Thomas A. Christensen, Arizona Research Laboratories, Division of Neurobiology, University of Arizona, 1040 East Fourth Street, Tucson, AZ 85721. E-mail: tc@neurobio.arizona.edu. DOI:10.1523/JNEUROSCI.3677-04.2004

Copyright $\odot 2004$ Society for Neuroscience $\quad$ 0270-6474/04/2411108-12\$15.00/0 odor molecules that evoke comparable spatial activation patterns? In an extension of previous models (Gelperin and Tank, 1990; Freeman, 1992), a proposed temporal coding mechanism could explain how molecularly similar odorants are discriminated in the insect AL (Stopfer et al., 1997; Laurent et al., 2001), but the well defined spatial aspects of the olfactory code are minimally represented in this model (Wehr and Laurent, 1996; Theunissen, 2003). Other studies in both vertebrates and invertebrates support an alternative model in which both the spatial and temporal aspects of the olfactory signals are inseparable components of the code (Spors and Grinvald, 2002; Christensen et al., 2003). To evaluate these hypotheses in greater detail, it is necessary to use a method that allows high-resolution monitoring of odor-evoked activity in both space and time.

Some studies have shown that mitral/tufted $(\mathrm{M} / \mathrm{T})$ cells or projection neurons (PNs) innervating the same glomerulus are more likely to show correlated firing in response to a stimulus (Buonviso and Chaput, 1990; Carlson et al., 2000; Lei et al., 2002; Christensen et al., 2003; Schoppa and Urban, 2003). Other studies have found, however, that the M/T cells innervating different glomeruli in the same region of the $\mathrm{OB}$ can also show strong correlations (Kashiwadani et al., 1999). Intraglomerular coactivity could play a role in amplifying the spatial representation of the specific chemosensory signal encoded in that glomerulus, whereas interglomerular synchrony could underlie a synthetic 
process that binds the different molecular features of the stimulus encoded in different information streams. Definitive coding roles for intraglomerular and interglomerular synchronization therefore have yet to be defined for any olfactory system.

To examine directly and simultaneously the roles of local and distributed network correlations in the neural representation of different olfactory stimuli at early stages of processing, we used a spatially defined array of 16 recording electrodes (Christensen et al., 2000; Daly et al., 2004b) to monitor both the spatial and temporal patterns of ensemble activity triggered by chemically different classes of odorants. Ensemble recordings revealed that each odor class evoked a different spatial focus of activity, but the correlated firing of action potentials within and between recording zones further distinguished the population codes for different odorants across the glomeruli of the main AL.

\section{Materials and Methods}

Experimental preparation. Adult male moths (Manduca sexta; Lepidoptera:Sphingidae) were reared in the laboratory on an artificial diet under a long-day (17/7 hr light/dark cycle) photoperiod. Animals were prepared for experiments 2-3 d after emergence, as described previously (Christensen et al., 2000). In preparation for recording, the moth was secured inside a plastic tube with dental wax, leaving the head and antennae exposed. The head was opened to expose the brain, and the tube was fixed to a recording platform attached to the vibration-isolation table. The preparation was oriented so that both ALs faced upward, and the tracheae and sheath overlying one AL were carefully removed with a pair of fine forceps. The brain was superfused slowly with physiological saline solution (in mM: $150 \mathrm{NaCl}, 3 \mathrm{CaCl}_{2}, 3 \mathrm{KCl}, 10 \mathrm{~N}$-tris[hydroxymethyl] methyl-2-aminoethanesulfonic acid buffer, and 25 sucrose, $\mathrm{pH}$ 6.9) for the duration of the experiment.

Olfactory stimulation. Olfactory stimuli were delivered to the preparation as reported previously (Christensen et al., 1993). Pulses of air from a constant air stream were diverted through a glass syringe containing a piece of filter paper, on which was deposited an odor compound $(10 \mu \mathrm{g}$ for plant-derived volatiles and $10 \mathrm{ng}$ for pheromone components). The odor stimulus was pulsed by means of a solenoid-activated valve controlled by an electronic stimulator (W-P Instruments, Sarasota, FL). In each experiment, the outlet of the stimulus syringe was positioned $\sim 2 \mathrm{~cm}$ from and orthogonal to the center of the antennal flagellum ipsilateral to the AL. Stimulus duration was $200 \mathrm{msec}$, and five pulses were separated by an interval of $2 \mathrm{sec}$ (supplemental material showing responses to longer stimulus durations is available at www.jneurosci.org).

Four classes of olfactory stimuli were used (see Fig. $1 A$ ): (1) aliphatic aldehydes: nonanal ( $n a l)$ and $E$-2-hexenal $(t 2 h) ;(2)$ aromatics: phenylacetaldehyde ( $p a a)$ and methyl salicylate (met); (3) monoterpenoids: ( \pm )linalool (lin), nerol (ner), ocimene (oci), myrcene (myr), and geraniol ( ger); and (4) sex pheromone components: E,Z-10,12-hexadecadienal (bombykal or bal; the primary component of the female sex pheromone), E, Z-11,13-pentadecadienal [c15; a mimic of a second component of the pheromone (Kaissling et al., 1989)], and the blend of the two components $(b l d)$. Some ensembles were not tested with $c 15$ and $b a l$ individually. The control solvent for plant volatiles was mineral oil (ctr) and for pheromone components was cyclohexane (can). All of the compounds tested are biologically important volatiles that Manduca encounters in its natural environment (Shields and Hildebrand, 2001; Fraser et al., 2003). In addition, an olfactory stimulus that the moth would not encounter naturally, cyclohexanone $(c y c)$, was included because olfactory conditioning studies have shown that Manduca learns to associate this odorant with sucrose reinforcement (Daly et al., 2001).

Ensemble recording and data analysis. The odor-evoked responses of 118 units were obtained in 12 male moths. Recordings were made with 16-channel silicon multielectrode recording arrays (MRAs) supplied by the Center for Neural Communication Technology at the University of Michigan (http://www.engin.umich.edu/facility/cnct/backind.html). To study the spatial distribution of odor-evoked activity, we selected an MRA design (a $4 \times 4$ array) that suits the dimensions of the AL in
Manduca (see Fig. 2A). These probes have four shanks spaced $125 \mu \mathrm{m}$ apart, each with four recording sites $50 \mu \mathrm{m}$ apart. The MRA was positioned under visual control using a stereo microscope. The four shanks were oriented in a line parallel to the antennal nerve, with the first shank inserted into the macroglomerular complex (MGC) (see Fig. 2B). The MRA was advanced slowly through the AL using a micromanipulator (Leica Microsystems, Bannockburn, IL) until the uppermost recording sites were just below the surface of the AL. In this manner, the four shanks of the MRA defined four zones of glomerular neuropil across the AL.

Ensemble activity was recorded simultaneously from the 16 channels of the MRA using two Lynx- 8 amplifiers (Neuralynx, Tucson, AZ). Spike data were extracted from the recorded signals and digitized at $20 \mathrm{kHz}$ per channel using Discovery acquisition software (Data Wave Technologies, Longmont, CO) and a 2821-G 16SE analog-to-digital board (Data Translation, Marlboro, MA) on a personal computer platform (Data Wave Technologies). Filter settings (typically $0.6-3 \mathrm{kHz}$ ) and system gains (typically 5,000-20,000) were software adjustable on each channel. Spikes were sorted using a clustering algorithm based on the method of principal components (PCs) (Off-line Sorter; Plexon, Dallas, TX). Only those clusters that were separated after statistical verification in threedimensional (PC1-PC3) space (multivariate ANOVA; $p<0.01$ ) were used for additional analysis ( $6-15$ units were isolated per ensemble; $n=$ 12 ensembles in as many animals). Spikes arising from the same unit were visible on adjacent recording sites, thus providing geometric information about the spatial origin of the signals. Each spike in each cluster was time-stamped, and these data were used to create raster plots (see Fig. 3) and to calculate peristimulus time histograms (PSTHs), interspike interval histograms, cross-correlograms, and rate histograms. All analyses were performed with Neuroexplorer (Nex Technologies, WinstonSalem, NC) using a bin width of $5 \mathrm{msec}$, unless noted otherwise.

Conditional response probability in single units. To assess the validity of the arbitrary grouping of chemical classes and to examine the response preference of each unit for certain olfactory stimuli, we calculated the conditional probability of response to all members of the stimulus set (see Fig. 1A) (Fletcher and Wilson, 2003). First, we grouped together all units that responded to a particular stimulus (e.g., reference stimulus $=$ linalool), then calculated the probability that this subset of units also responded to the remaining members of the stimulus set (comparison stimuli). For example, the probability that linalool-responsive units also responded to nerol was calculated as follows:

$$
P_{\text {ner/lin }}=N_{\text {ner/lin }} / N_{\text {lin }}
$$

where $N_{\text {ner/lin }}$ was the number of nerol-responsive units within the pool of linalool-responsive units, and $N_{\text {lin }}$ was the total number of linaloolresponsive units. If two odorants activated the same pool of units, the conditional probability for the two odorants would be 1.0. To summarize results for the entire data set, the conditional probability scores for all olfactory stimuli were color-coded and displayed in a response-probability matrix, as shown in Figure 4.

Spatial distribution of odor-evoked activity. Sorted units were arranged according to which of the four AL zones (I-IV) defined by the MRA yielded each recording (see Figs. 2 A, 3). For each unit sorted, PSTHs were generated for all responses to each odor stimulus and the control (solvent only) stimulus. The response window was defined as the $500 \mathrm{msec}$ period beginning at the onset of the $200 \mathrm{msec}$ stimulus pulse. A unit was considered to be responsive if its control-subtracted PSTH was above (excitatory) or below (inhibitory) the $95 \%$ confidence limits derived from a cumulative sum (CUMSUM) test based on the Monte Carlo method (Ushiba et al., 2002). Inspection of the entire 118 unit data set revealed that a $500 \mathrm{msec}$ window was sufficient to capture all of the $1000+$ stimulus-evoked responses recorded. We quantified the controlcorrected response for every unit by calculating a response index (RI) similar to that used to quantify the uptake of 2-deoxyglucose in the rat OB (Johnson et al., 2002). RI values reflect the deviation from the mean response of all units across all odors in one ensemble, as follows:

$$
\mathrm{RI}=\left(R_{\text {odor }}-R_{\mathrm{m}}\right) / \mathrm{SD},
$$

where $R_{\text {odor }}$ is the number of spikes evoked by the test odor minus the number evoked by the control stimulus, $R_{\mathrm{m}}$ is the mean response, and $\mathrm{SD}$ 
A

\section{Aliphatic Aldehydes (AA)}

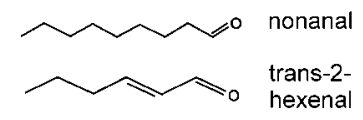

Monoterpenoids (MO)

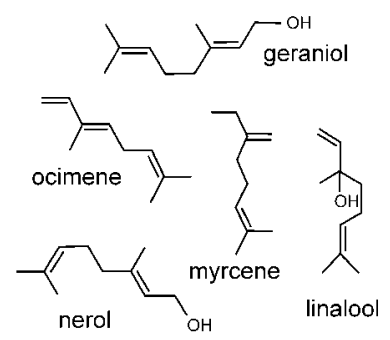

B

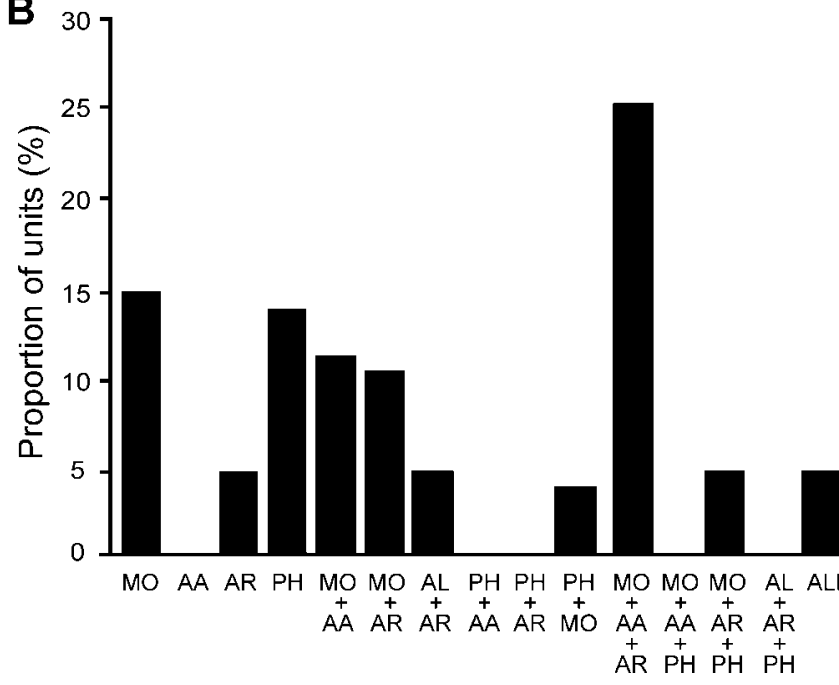

Figure 1. The panel of olfactory stimuli. A, Four major odorant classes, conspecific sex pheromone components (Kaissling et al., 1989) or host plant volatiles for M. sexta (Fraser et al., 2003), were used: aliphatic aldehydes (AA): nonanal and E-2-hexenal; aromatics (AR): phenylacetaldehyde and methyl salicylate; monoterpenoids (MO): ( \pm )linalool, nerol, ocimene, myrcene, and geraniol; pheromonal components (PH): E,Z-10,12-hexadecadienal, E, Z-11,13pentadecadienal [a stable mimic of the second major pheromone component (Kaissling et al., 1989)], and the blend of $b a l$ and (15. Another tested volatile that is not naturally occurring but that Manduca can learn to associate with sucrose reinforcement is the simple ketone (SK) cyclohexanone. $B$, Percentage of units that exhibited selective responses to $M 0, A A, A R$, or PH volatiles and nonselective responses to two or more of these stimulus classes.

is the $\mathrm{SD}$ across the data matrix. The RI values for the nonresponsive units fell between -2 and +2 , based on the CUMSUM test. Given that $>50 \%$ (on average) of recorded units in each ensemble were unresponsive, $R_{\mathrm{m}}$ approximated the background activity level, and thus negative values of the RI indicated response suppression. The RI values for all units were color-coded and arranged as an activity matrix with each row representing the ensemble response to a different odor stimulus (see Fig. 5). The RI had a range from -3.0 SDs (strongly inhibited units; cool colors) to +3.0 SDs (strongly excited units; warm colors).

Calculating response profiles and profile similarities (profile similarity index) between units. Variation across the responses to the different test stimuli was summarized as a color-coded correlation matrix for each unit (see Fig. $6 C, D$ ). This matrix was generated by calculating the 55 pairwise correlations between the PSTHs for each of the 11 stimuli and then translating the correlation coefficients into a color scale. To increase statistical efficiency (Kass et al., 2003), we applied a Gaussian filter (bandwidth of three bins) to the control-corrected PSTHs, each covering a time window starting at $200 \mathrm{msec}$ before and ending at $1.5 \mathrm{sec}$ after stimulus onset. Using a $5 \mathrm{msec}$ bin width, each PSTH generated a vector containing 340 elements. Pairwise correlations of these vectors were then performed to obtain the 55 values for each matrix. We define the matrix as the overall "signature" of the neuron or a response profile that summarizes the similarity between the responses of the neuron to each pair of odor stimuli. The correlation coefficients represented on the color scale in Figure 6 are therefore a measure of response similarity, not response intensity as in Figure 5A. Next, to compare the response profiles between any two units, the two signature matrices were transformed into separate vectors, and the correlation coefficient between the two vectors was calculated (this coefficient was calculated for all pairs of units). We define this coefficient as the profile similarity index (PSI), which ranged from 0.0 (no similarity) to 1.0 (indistinguishable signatures) for each pair of units (see Figs. 6F, 7). A PSI near 1.0 indicated that the two neurons were likely receiving common input.

Measuring stimulus-evoked coactivity between units. To calculate the temporal relationship between each pair of units, we used a crosscorrelation analysis using the following formula:

$$
C_{\%}=\frac{[C E]_{\mathrm{RAW}}-[C E]_{\mathrm{SHUFFLED}}}{N_{1}(T)+N_{2}(T)} \times 100 \%,
$$

where $[C E]_{\text {RAW }}$ is the number of coincident events in the $5 \mathrm{msec}$ crosscorrelogram peak centered around $t=0$, and $[C E]_{\text {SHUFFLED }}$ is the number of coincident events after trial shuffling (shift predictor method) to correct for coincidences attributable to chance and an increased firing rate (Aertsen et al., 1989). The corrected correlograms were calculated by averaging over four trial shifts and subtracting the result from the raw correlogram. $T$ is the total response time over which spikes were counted (500 msec), and $N_{1}$ and $N_{2}$ are the total number of spikes recorded from units 1 and 2 during time $T$. The coactivity index $\left(C_{\%}\right)$ therefore reflects the percentage of coincident spikes relative to the total number of spikes recorded from the two neurons (see Fig. $6 F$ ). All calculations were implemented in Matlab 6.5 (The Mathworks, Natick, MA) or Neuroexplorer (Nex Technologies).

To visualize the odor-dependent coactivity pattern within an ensemble, we arranged individual units in a circular array similar to the "connectivity matrix" used to describe ensemble patterns in the hippocampus (Wilson and McNaughton, 1994). Each pair of units was connected with a line that depicted the magnitude of the correlation $\left(C_{\%}\right)$ : values from $10-19 \%$, dashed line; values $\geq 20 \%$, solid line (see Fig. $8 B, C$ ). In addition, each unit was color-coded to reflect its response magnitude, and these values were used to make a direct comparison between the spatial and temporal activity patterns evoked by different odorants (see Fig. 8C). To measure the ensemble-wide similarity between coactivity patterns evoked by different olfactory stimuli, we transformed the coactivity patterns composed of positive $C_{\%}$ values for all unit pairs in each ensemble into one-dimensional vectors (one for each odorant) and then calculated the correlation coefficient between the two vectors. This transformation is shown for one ensemble in Figure $8 D$ and repeated for the entire data set in Figure $8 E$. Population data were calculated only within, not between, ensembles.

Histological identification of recording probes. To examine the precise location of the recording probes, the brain was excised and immersed in $1-2 \%$ glutaraldehyde in $0.1 \mathrm{~m}$ phosphate buffer to increase tissue contrast and facilitate locating probe tracks. Brains were fixed for at least $1 \mathrm{hr}$, then dehydrated with a graded ethanol series, cleared in methyl salicylate, and finally imaged as whole mounts with a laser-scanning confocal microscope (Nikon PCM 2000 equipped with a $457 \mathrm{~nm}$ argon laser). Optical sections were $5 \mu \mathrm{m}$, and this method reliably revealed the tracks of the four MRA shanks in the AL without the need for tissue staining (see Fig. $2 B$ ). The identification of individual glomeruli is an important prerequisite for assigning functional significance to a given glomerulus, but this is beyond the scope of this study. 

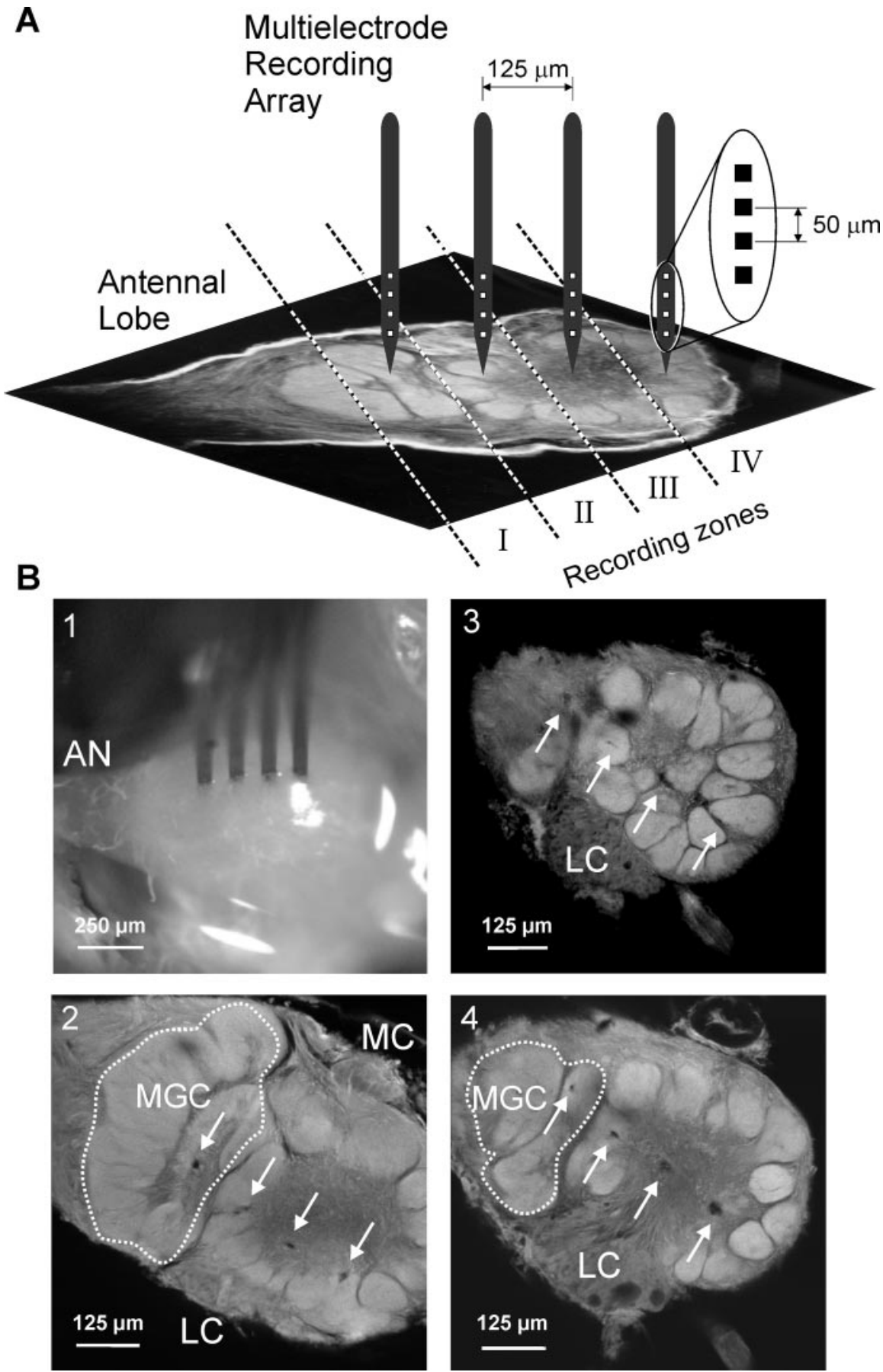

Figure 2. Positioning the recording array in the moth $\mathrm{AL}$. $A$, The 16 channel MRA has four shanks with four electrodes spaced evenly along each shank. The dimensions of the MRA were selected so that each shank would record activity in one of four broad zones (I-IV) of processing neuropil in the AL. $B$, The MRAs were placed in the same zonal arrangement in the AL from animal to animal. $B 1$, The antennal nerve (AN) was used as a landmark to help align the MRA in the AL; the leftmost shank was always inserted into the MGC. B2, A single optical section from a confocal stack showing the lesions (white arrows) left by the four shanks of the MRA. In a different preparation, a section near the AL surface (B3) and one $25 \mu \mathrm{m}$ deeper (B4) show the trajectories of the shanks where they penetrated through the AL neuropil. A comparison of plates 2 and 4 illustrates the variation across animals in positioning the MRA shanks, showing that the shanks can be placed repeatedly within the same AL zones, as schematized in $A$.

\section{Results}

AL ensembles consist of functionally diverse cell types encoding olfactory signals

Results were based on a sample of 118 units recorded from 12 ensembles ( 1 ensemble per animal). After completion of testing with the full set of odorants (Fig. 1A), we found that approximately one-third of the units $(34 \%)$ was selectively responsive to only one of the stimulus classes tested, whereas the remaining units were clearly more broadly responsive across classes (Fig. $1 B$ ). Variation among cells in response selectivity to different odor classes suggests that all ensembles likely consisted of mixed populations of local interneurons (LNs) and PNs (Christensen et al., 1993, 1998). The four shanks of the MRA furthermore spanned a total of $375 \mu \mathrm{m}$, making it possible to record stimulusevoked ensemble activity from multiple sites across the AL (Fig. 2A). The dimensions of and spacing between the shanks permitted recording from four distinct glomerular zones (zones I-IV) in the AL (Fig. 2A). Although these zones are not histologically distinct, they are occupied by nonoverlapping subsets of glomeruli (Rospars and Hildebrand, 2000), and the MRA design allowed us to sample the same four AL zones consistently across animals (Fig. $2 B$ ).

\section{Conditional response probabilities}

We tested the responsiveness of neural ensembles (Fig. 3) to olfactory stimuli that were grouped into four broad chemical classes: sex pheromone components, monoterpenoids, aromatics, and aliphatics (Fig. 1). We first determined the effective stimuli for each of the 118 recorded units using the CUMSUM test (see Materials and Methods), identified the subset of units that responded to a particular odor (Fig. 4, reference stimulus), and then calculated the probability that each unit also responded to one or more of the other odor compounds in the stimulus set (Fig. 4 , comparison stimulus). In the group of units that showed an excitatory response to lin, for example, the majority showed similar responses to ner and oci (also monoterpenoids), but these units were less likely to respond to odorants belonging to the other chemical classes (Fig. 4). A similar pattern was observed for the subset of units that responded to the aromatic stimulus paa; these units were more likely to respond to met (another aromatic) than to any other odorants in the stimulus set (Fig. 4). The clustering of responses into discrete classes was less evident for the aliphaticbest units. For instance, as expected, nalbest units were also responsive to $t 2 h$ (another aliphatic molecule), but they responded also to lin, oci, and myr (monoterpenoids) as well as less strongly to the aromatic paa and to the simple ketone $c y c$ (Fig. 4). 
Each chemical class evokes a spatially distinct pattern of excitation and inhibition

Based on the results of the conditional probability analysis, we predicted that some members of the same odor class would produce similar spatial representations because they activated mostly overlapping populations of units. This was indeed the case, as shown in Figure 5. However, although patterns within a class were similar, each of the four chemical classes was represented by a distinct zonal pattern in the AL. At one extreme, as expected from our previous results, the greatest excitatory RI values for sex pheromone-selective units were focused in the MGC, located in zone I (Fig. 5A). In accordance with recent imaging data (Hansson et al., 2003), a zonal focus of activity (indicated in bold below) was also observed for the nonpheromonal odorants, but these responses were more widely distributed across the AL. The spatial ranking of excitatory responses to the aromatics met and paa showed the following pattern: zone IV > III > II; for monoterpenoids, zone III $>$ II $>$ IV $\geq$ I; for aliphatics, zone II $>$ IV $>$ III (Fig. 5B). The pattern for the simple ketone $c y c$ was difficult to distinguish from that evoked by the monoterpenoids (Fig. 5B).

A substantial body of evidence from previous studies in widely different species shows that glomerular activity patterns are also modulated by inhibitory LNs (Christensen and White, 2000; Lei et al., 2002; Sachse and Galizia, 2002, 2003; Aungst et al., 2003). When this critical coding dimension was also considered as a component of the ensemble representation, it became evident that inhibitory interactions between recording zones further broadened the spatial extent of the ensemble representations for most olfactory stimuli (Figs. 3, 5). Both the narrowly and broadly responsive ensemble units displayed inhibitory responses to the tested volatiles. For example, in the ensemble illustrated in Figure 3, one unit in zone II (II-2) and another unit in zone IV (IV-2) were inhibited by lin, although the focus of excitatory activity was in zone III. Other units shown in Figure 6, $A$ and $B$, were excited by three monoterpenoids (lin, ner, and ger) but were inhibited by all other members of the stimulus set. These findings support a role for lateral-inhibitory interactions between glomeruli in shaping the representational maps for different olfactory stimuli. These lateral-inhibitory interactions also appear to operate between the two major subsystems in the male's AL. One such interaction is illustrated by the units found in zones II-IV that were strongly inhibited by pheromonal input, which is processed initially in the MGC located several hundred micrometers away in zone I. When we analyzed the inhibitory response fields across all animals and plotted all values of RI $\leq$ -2.0 SDs (Fig. $5 B$ ), we found that unlike their excitatory fields, the inhibitory representations of all stimuli were widely distributed throughout the AL. Inhibition also spread from the main AL, as shown by significant inhibitory responses in the MGC in response to the monoterpenoid oci and the two aromatics (Fig. 5B).

\section{Temporal patterning underlies the spatial maps}

An important advantage of the ensemble-recording technique is that stimulus-dependent neural interactions can be examined in both the temporal and spatial domains (Brown et al., 2004). Increasing evidence indicates that specific temporal relationships among AL neurons also may be used by the brain to represent the different features of an olfactory stimulus (Christensen et al., 2000; Laurent et al., 2001; Lei et al., 2002; Müller et al., 2002; Sachse and Galizia, 2002; Spors and Grinvald, 2002). For example, MGC output neurons tuned to the same component of the female's sex pheromone in Manduca exhibit correlated activity in response to this component (Lei et al., 2002). It is not known, however, whether similarity in odor tuning between units responsive to general odorants can also predict temporal correlations between them. To examine the relationship between response tuning and correlated spike activity, we first compared the responses from each unit across the entire set of olfactory stimuli (Fig. 6A, B) (see Materials and Methods). This analysis revealed a complex pattern of both high and low correlations $(-1.0$ to +1.0 ) between the responses to the different odorants. In the examples shown in Figure 6, $C$ and $D$, the signature matrices computed for the two units revealed the greatest similarity between the responses to the monoterpenoids lin, ner, and ger (solid circles). We next calculated the correlation coefficient between the two signature matrices to obtain a PSI (see Materials and Methods) for this pair of units, and similar calculations were performed for all of the remaining pairs of units in each ensem- 


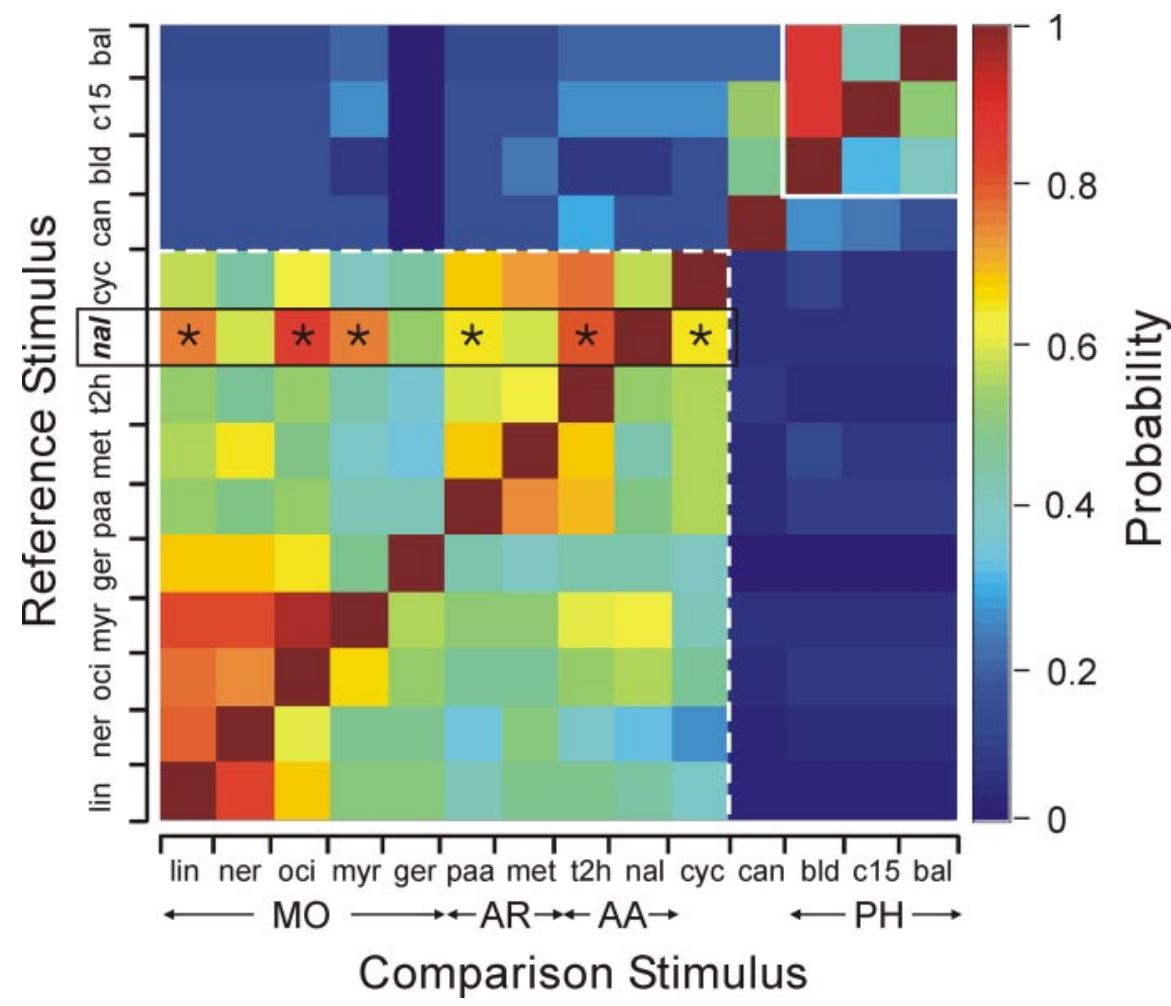

Figure 4. Conditional response probabilities. Units that responded to one member of an odor class were likely to show similar responses to other members of the same class. The color matrix summarizes the conditional response probabilities calculated for all units in the data set $(n=118)$. The diagonal of the matrix shows the conditional probability $(p=1.0)$ for each stimulus against itself. Note the separation of the data into two clusters: one for plant odorants (dashed outline) and another for pheromones (solid outline). Units that responded to monoterpenes $(\mathrm{MO})$ showed the least variation across odorants. The conditional response probabilities for all units responsive to $n a l$ are outlined in black. The other stimulus compounds with strong response probabilities ( $p>0.6$ ) are indicated by asterisks. AR, Aromatics; AA, aliphatic aldehydes; $\mathrm{PH}$, pheromonal components.

either 250 or $375 \mu \mathrm{m}$ apart. We then calculated the mean $C_{\%}$ values for all pairedunit responses to all odorants and separated the results according to intershank distance. The data show that the mean values of $C_{\%}$ across all odor stimuli did not exceed $11.6 \%$ ( $n=12$ moths) (Table 1$)$. The total number of unit pairs recorded on the same shank (0 $\mu \mathrm{m}$ separation) was 173, and for intershank distances of 125 , 250 , and $375 \mu \mathrm{m}$, the numbers were 246 , 77 , and 39 , respectively. In $50 \%$ of the moths, the coactivity index was not significantly greater at any of the intershank distances, whereas in the remaining 50\% of the moths, units recorded on adjacent shanks (125 $\mu \mathrm{m}$ separation) exhibited significantly greater synchrony (Table 1 ). Collectively, these results indicate that in response to an olfactory stimulus, a relatively small subset of similarly responsive AL neurons exhibits temporal correlations both within and between glomerular zones. On average, the greatest number of synchronous firing events occurred between neighboring zones of glomerular neuropil, but only half of the preparations showed statistical significance (Fig. 7D, Table 1).

We also found that although the ensemble patterns evoked by different members of the same odor class were spatially overlapping, the pattern of network coactivity evoked by each individual odorant was nevertheless distinct. Figure 8 illus-

ble. We then used the time-stamped spike data to calculate stimulus-evoked temporal correlations between each pair of units in each ensemble ( $C_{\%}$; see Materials and Methods). A plot of the PSI versus $C_{\%}$ for the responses to $\operatorname{lin}$ in one ensemble is shown in Figure $6 F$, revealing a positive relationship between synchronous spiking activity and similarity of response profile between units. The same relationship was observed after combining the data for all ensembles: as the $C_{\%}$ value between units increased, the binned histogram data again showed a progressive shift toward higher PSI values (Fig. $7 B$ ). Plotting the mean histogram values for lin (thick line) and all of the remaining odorants revealed a similarly strong correlation between the PSI and synchronous activity for all stimuli (Fig. 7C).

\section{Integrating activity patterns in space and time}

Up to this point, we have examined the relationship between stimulus tuning and coactivity in single pairs of AL neurons. From the time this coding issue was first raised $>50$ years ago, however, it remains unresolved whether the discrimination of the spatially overlapping ensemble representations evoked by different olfactory stimuli may be further enhanced by the stimulusdependent pattern of correlated firing across the entire coding ensemble. To address this critical issue, we looked at how the pattern of correlated firing across ensembles is zonally distributed in the AL by grouping all unit pairs according to the intershank distance between the two recordings (Fig. 7D). In our experiments, units recorded on adjacent shanks were separated by $125 \mu \mathrm{m}$, whereas those pairs recorded between other shanks were trates the spatial and temporal relationships between two chemically related monoterpenoids, $l i n$ and ner. These odorants elicited very similar responses in single units (Fig. 8A) as well as heavily overlapping spatial response patterns across the complete ensemble of 10 units. A strong correlation between the spatial patterns $(r=0.93)$ showed that the spatial characteristics of the responses to these two stimuli were nearly indistinguishable (Fig. $8 C$ ). However, analysis of the stimulus-evoked pattern of synchronous firing across units in the ensemble revealed a very different result. We found that the value of $C_{\%}$ between unit pairs responding to lin and ner ranged from 0 to $\sim 35 \%$ (Fig. $8 B$ ). Furthermore, the overall pattern of coactivity was distinctly different for the two stimuli (Fig. 8C). For responses to both lin and ner, the strongest temporal correlations occurred between units II-2 and III-3, but another pair (IV-1 and IV-2) showed a stronger correlation $\left(C_{\%}=24 \%\right)$ in response to ner. Weaker correlations between 10 and $20 \%$ were also found (Fig. $8 C$, dashed lines), and the patterns of these correlations were clearly distinct for the two odorants. An unexpected finding was that strong correlations sometimes occurred between nonresponsive units (i.e., $-2<\mathrm{RI}<+2$; e.g., units IV-1 and IV-2 after stimulation with ner), suggesting that even units that are statistically unresponsive individually may nevertheless participate in the coding ensemble through temporal coordination of their firing with other units (Fig. 8C).

To measure the similarity between coactivity patterns, we again transformed the patterns composed of all unit pairs $(n=$ 45 ) into vectors (one vector for each stimulus) and then calcu- 
lated the correlation coefficient between the two vectors. The result $(r=0.79)$ was $15 \%$ lower than that calculated for the spatial response pattern alone, indicating that the ensemble-wide patterns of synchronous firing for the two stimuli were more distinct than the spatial patterns alone (Fig. 8D). These differences are statistically significant even at the population level ( $p<0.05$; Mann-Whitney $U$ test; $n=535$ pairs of units across 12 animals) (Fig. $8 E$ ). Collectively, these results demonstrate that the spatial activity patterns evoked by different olfactory stimuli within a class can overlap considerably across the AL, potentially confounding the discrimination of these stimuli. When the pattern of coincident firing is also taken into account, however, a distinctly different ensemble pattern emerges for each stimulus.

\section{Discussion}

Odor representations across olfactory glomeruli constitute a complex mosaic of excitatory and inhibitory activity, and neural-ensemble recordings provide a uniquely powerful tool to examine the dynamic and distributed character of these odor-dependent network representations in the brain (Christensen et al., 2000; Lehmkuhle et al., 2003; Stopfer et al., 2003; Daly et al., 2004a,b). Our ensemble data show that each of the plant-derived odorants that we tested evoked a stereotyped and combinatorial pattern of spiking activity across the glomeruli in the moth AL. These patterns did, however, show considerable spatial overlap, and some members of the same chemical class produced highly overlapping activity patterns (Fig. 5). Consistent with molecular evidence that olfactory neurons expressing homologous odorant receptors project to neighboring glomeruli (Tsuboi et al., 1999), support for spatially organized odor representations comes from numerous studies in widely differing species (for review, see Christensen and Hildebrand, 2002; Korsching, 2002; Keller and Vosshall, 2003). Our population data now provide direct evidence that the representations of nonpheromonal olfactory stimuli involve a combinatorial pattern of both excitatory and inhibitory responses distributed across each coding ensemble. Moreover, we found that approximately one-third of the neurons we sampled showed high selectivity (Fig. $1 B$ ) and were localized to specific zones of the AL neuropil (Fig. 5). The spatial activity patterns derived from our MRA data also match closely with previous functional mapping studies in the same species. Optical imaging using calcium-sensitive dyes showed that the spatial patterns triggered by aromatic volatiles were localized to the most medial portion of the AL (Hansson et al., 2003), and the same region in our study (zone IV) represents the excitatory activity focus for the aromatics met and paa (Fig. 5). A similar corresponthe AL.

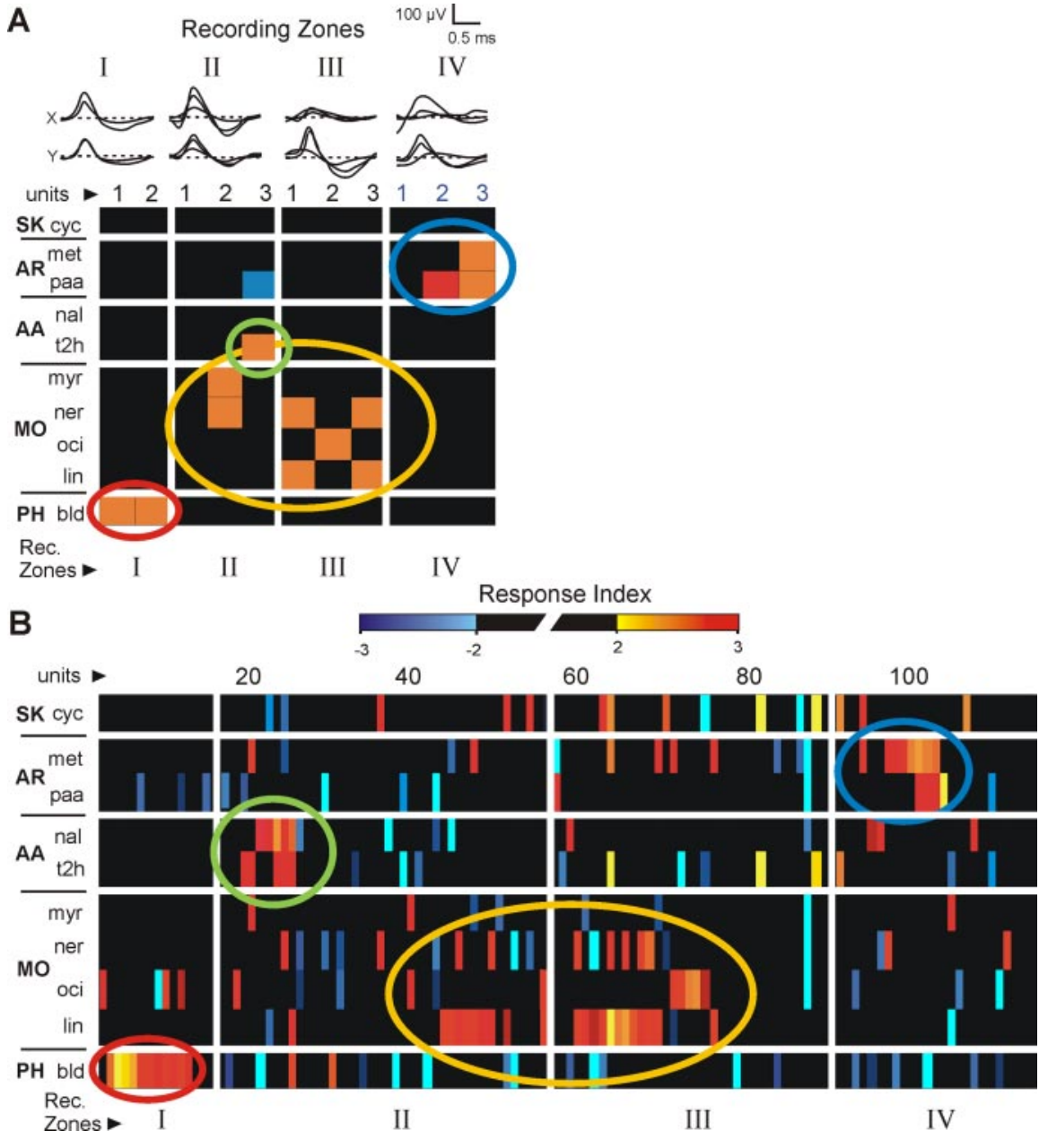

Figure 5. Ensemble representations in space. A, Spatial distribution of activity evoked across the AL in one ensemble, summarizing the population response to a diverse set of odorants. The top plots show the averaged spike waveforms from two (shank I) or three (shanks II-IV) units recorded simultaneously. All units were visible on adjacent recording sites ( $X$ and $Y$ ), thus providing geometric information about the spatial origin of the signals. The bottom plot is the color-coded response matrix from the 11 unit ensemble recorded across all shanks (columns) after stimulation with the different odorants (rows). Four major stimulus classes mones (PH) (c15, bal, bld), plus a simple ketone (SK) (cyc). Weak or null responses are not shown. Only the excitatory responses with an $\mathrm{RI} \geq 2.0 \mathrm{SD}$ s and inhibitory responses with an $\mathrm{RI} \leq-2.0 \mathrm{SD}$ s are shown for clarity (see color scale shown at the top of $B$ ). spatial mapping of excitatory and inhibitory responses for the entire data set ( $n=118$ units). For excitatory responses, sex (yellow outline). In contrast to the focal organization of the excitatory response fields, inhibitory responses were distributed across

dence between imaging and ensemble-recording data also exists for excitatory responses to monoterpenoids (e.g., lin) (Fig. 5). These two independent methods therefore confirm for Manduca that different classes of odorants are represented as overlapping but distinct spatial patterns of activity in the AL glomeruli. Accumulating evidence from the vertebrate $\mathrm{OB}$ (Rubin and Katz, 1999; Fuss and Korsching, 2001; Nikonov and Caprio, 2001) and insect AL (Gao et al., 2000; Vosshall et al., 2000; Galizia and Menzel, 2001) also supports this combinatorial spatial model.

From an information-processing perspective, chemotopic mapping of olfactory glomeruli offers the organism at least three key advantages: (1) the convergence of a large population of functionally equivalent olfactory receptor cells onto a single glomerulus reduces the detection threshold for weak signals above a noisy background; (2) a combinatorial glomerular map increases 
A

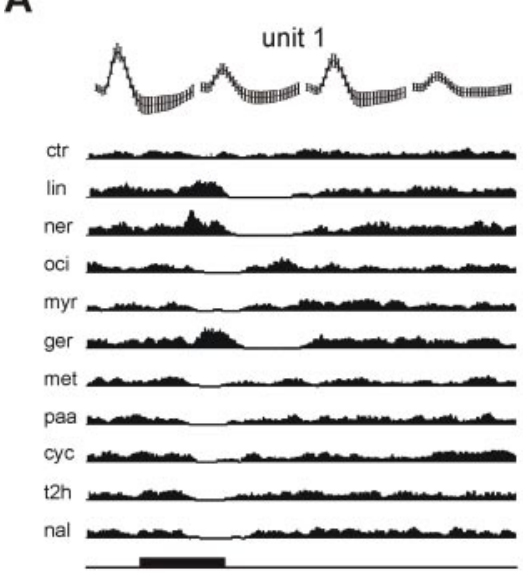

C

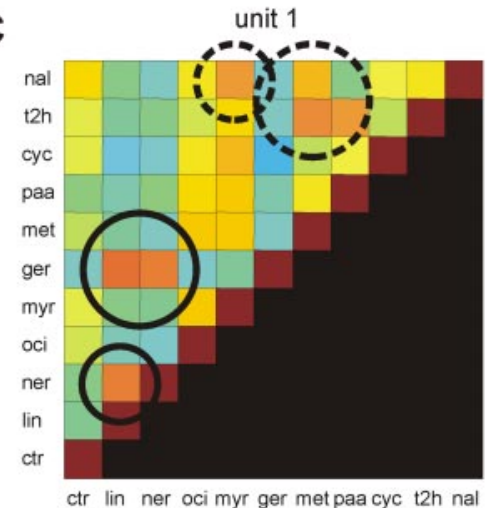

E

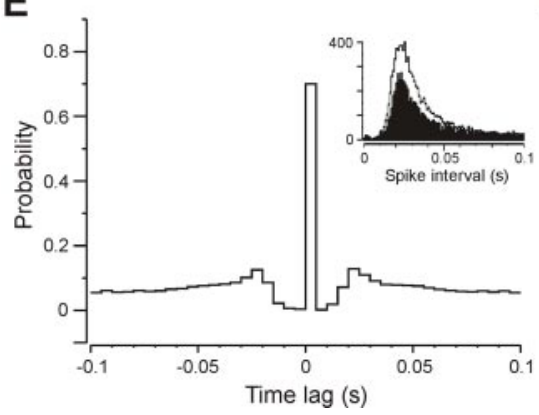

B
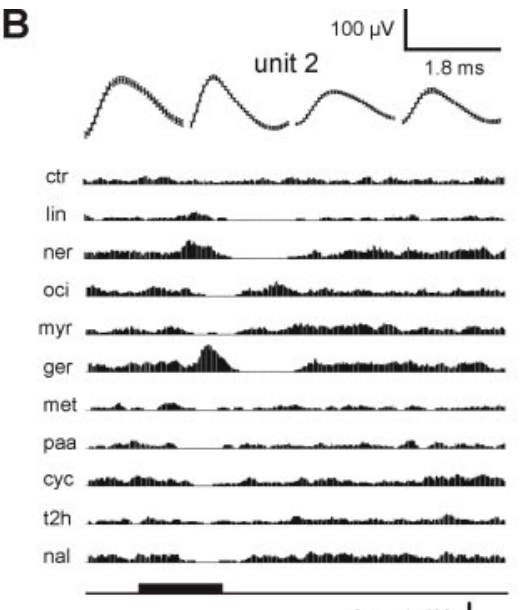

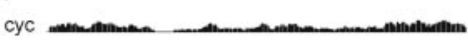

t2h

nal

\section{D}

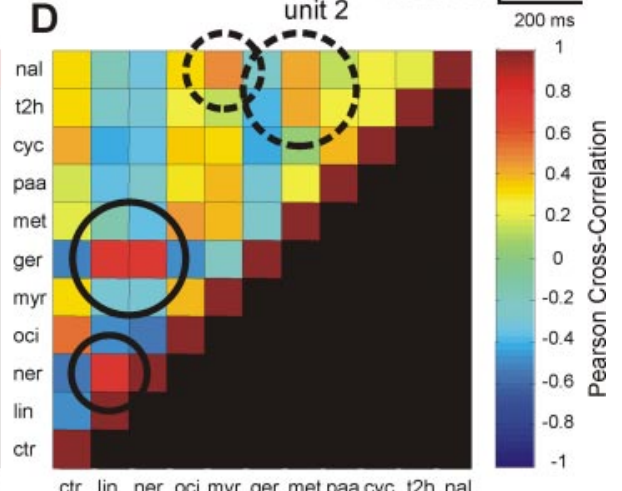

F

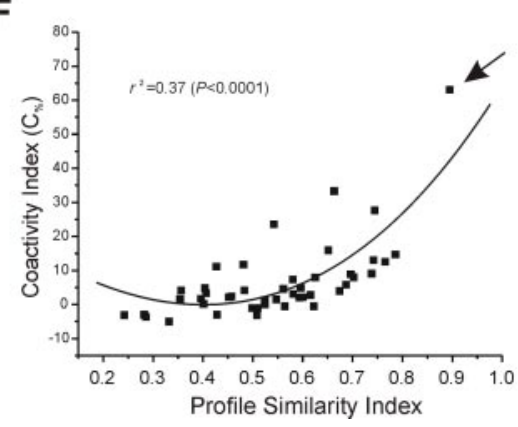

Figure 6. Ensemble representations in time. $A, B$, Two of the nine units in this ensemble were easily distinguishable by spike shape [the top traces show the averaged waveforms ( \pm SD) recorded on the 4 adjacent sites of shank II]. Although their spike shapes were different, the stimulus-response profiles of these two units were very similar. Spiking in both units (PSTHs) was increased by three monoterpenoids (lin, ner, and ger) but suppressed by the other seven stimulus compounds. C, D, To quantify the variability across responses, a color-coded correlation matrix or signature response profile was generated for each unit. Each matrix reflects the response variation between every pair of volatiles in the entire stimulus panel and thus serves as a signature of all of the responses of a single unit to the same panel of stimuli. This analysis revealed complex patterns of both high (warm colors) and low (cool colors) correlations between responses. The greatest correlations between excitatory responses (solid circles) and inhibitory responses (dashed circles) are indicated. Note also that the matrices for these two units are nearly identical, leading to a high PSI between the two units (see Materials and Methods and $F$ ). E, (ross-correlation analysis ( 5 msec bin width) also revealed a strong temporal relationship between these two units. The linalool-evoked correlation after trial shuffling to correct for the elevated firing rate is shown (see Materials and Methods). Interspike interval histograms for the two units are shown in the inset ( $y$-axis, counts at each interval). F, The PSI was defined as the correlation coefficient between the response profile vectors for a pair of units (as in $\left(\right.$ and $D$ ). The exponential curve illustrates the relationship between response similarity and unit coactivity $\left(C_{\%}\right)$ for all pairs of units in the ensemble. For all 36 pairwise combinations, the PSI ranged from 0.24 to 0.90 , revealing that pairs of units that responded similarly across the stimulus set were also positively correlated in time. The strongest relationship was found between the two units described in (and D (arrow). The coefficient of determination $\left(r^{2}\right)$ indicates that $37 \%$ of the total variation can be explained by this relationship. See Figure 7 for pooled data from all ensembles ( $n=535$ unit pairs).

exponentially the coding capacity of an olfactory network, especially one like the moth's AL that consists of relatively few glomeruli; and (3) the hardwired connection between different levels of processing in the olfactory pathway increases the speed of information transmission. Experimental evidence that structurally similar stimulus compounds can be discriminated through spatial coding is growing steadily (Rubin and Katz, 1999; Johnson and Leon, 2000a,b; Uchida et al., 2000; Inaki et al., 2002; Meijerink et al., 2003; Xu et al., 2003). Moreover, in Drosophila, the chemotopic arrangement of glomeruli is also preserved at the next stage of processing in the protocerebrum (Marin et al., 2002; Wong et al., 2002).

The PN activity map also undergoes considerable transformation as a consequence of inhibition mediated by GABAergic LNs. Inhibitory, spiking LNs interconnect many, if not all, glomeruli in the moth's AL (Christensen et al., 1993), and both ultrastructural and electrophysiological data indicate that LNs receive direct synaptic input from receptor cells (Tolbert and Hildebrand, 1981; Christensen et al., 1993). Inputs to the same LN from functionally different subpopulations of receptors therefore converge on different dendritic targets on the LN as defined by their specific branching patterns in the glomeruli. The spread of inhibition from wide-field LNs to PNs contributes substantially to the spatially distributed response of the PN ensemble before this information is relayed to the protocerebrum. Morphometric modeling studies indicate that the lateral interactions mediated by LNs will be stronger between adjacent glomerular zones (Christensen et al., 2001), but longer-distance interactions are also possible. In the present study, we provide experimental evidence to support the computer models: lateral inhibition, triggered by sex pheromonal input to zone I, can spread across the entire AL to suppress the firing of units in zones II, III, and even IV (Fig. 5B). In the same manner, stimulation with the aromatic compound paa produced a focus of excitatory activity in zone IV, but the same odorant evoked inhibitory responses in the remaining three zones, including zone I (Fig. 5B). Monoterpenoid responses revealed a "center-surround"-type organization, with an excitatory center in zones II and III and inhibitory surrounds in zones I and IV (Fig. 5B). Other inhibited units, however, were found in the same zones as the activated units. These data therefore provide direct evidence for both interzonal and intrazonal inhibition in that inhibition can spread long distances across the AL to influence the activity of distant glomeruli, but it can also function locally, providing inhibitory regulation to PNs within the activated glomerulus or glomeruli. Our data are furthermore consistent with recent imaging studies in other insects (Ng et al., 2002; Sachse and Galizia, 
2002). Both local and global inhibition has been reported in the honeybee's AL, where these two inhibitory systems may use different neurotransmitters that operate in concert during the response to odor (Sachse and Galizia, 2002). Global inhibition may serve to enhance contrast in the odor representation by suppressing the activity of glomeruli in the regions surrounding the central focus of excitatory activity (Figs. 3, 5) (Yokoi et al., 1995; Aungst et al., 2003). In contrast, local inhibition likely serves to enhance the timing precision of spiking between PNs innervating the same glomerulus (Lei et al., 2002) (see below).

Long-term ensemble recording allows testing of multiple and diverse olfactory stimuli, and our results demonstrate that each individual AL unit displays a unique signature response across a wide range of different volatiles, thus confirming that individual glomeruli participate in the coding of multiple odorants (Fig. 6C,D). We extended this observation by demonstrating that the strength of synchronous firing between units also increased as a function of similarity in their response profiles (Figs. 6F, 7C). Likewise, none of the pairs with low response similarity displayed significant coactivity. This result agrees with an increasing number of studies showing that specific temporal relationships among functionally defined neurons may be used by the brain to recognize and discriminate different features of an olfactory stimulus, although the mechanisms underlying these temporal relationships may vary in different olfactory systems (Christensen et al., 2000, 2003; Laurent et al., 2001; Lei et al., 2002; Sachse and Galizia, 2002). Manduca PNs, unlike those in locusts (Laurent et al., 2001), exhibit transient synchronization in response to olfactory stimulation, not involving fast oscillations (Christensen et al., 2003; Daly et al., 2004a,b).

Importantly, the patterns of coactivity need not involve many spikes from the units in the coding ensemble. In fact, our sample of 535 within-ensemble pairs of recorded units revealed that, on average, $<12 \%$ of the odor-evoked spikes in each pair were temporally correlated within 5 msec (Table 1). This is consistent with the transient nature of the coordinated ensemble response to brief olfactory stimuli (Fig. 3). It is also important to note that this stimulus condition is closely analogous to the situation in breathing animals. Numerous studies in mammals have shown, for example, that odors evoke respiration-coupled activity in M/T cells (for review, see Sobel and Tank, 1993; Cang and Isaacson, 2003; Margrie and Schaefer, 2003). Moreover, the brief responses of these vertebrate
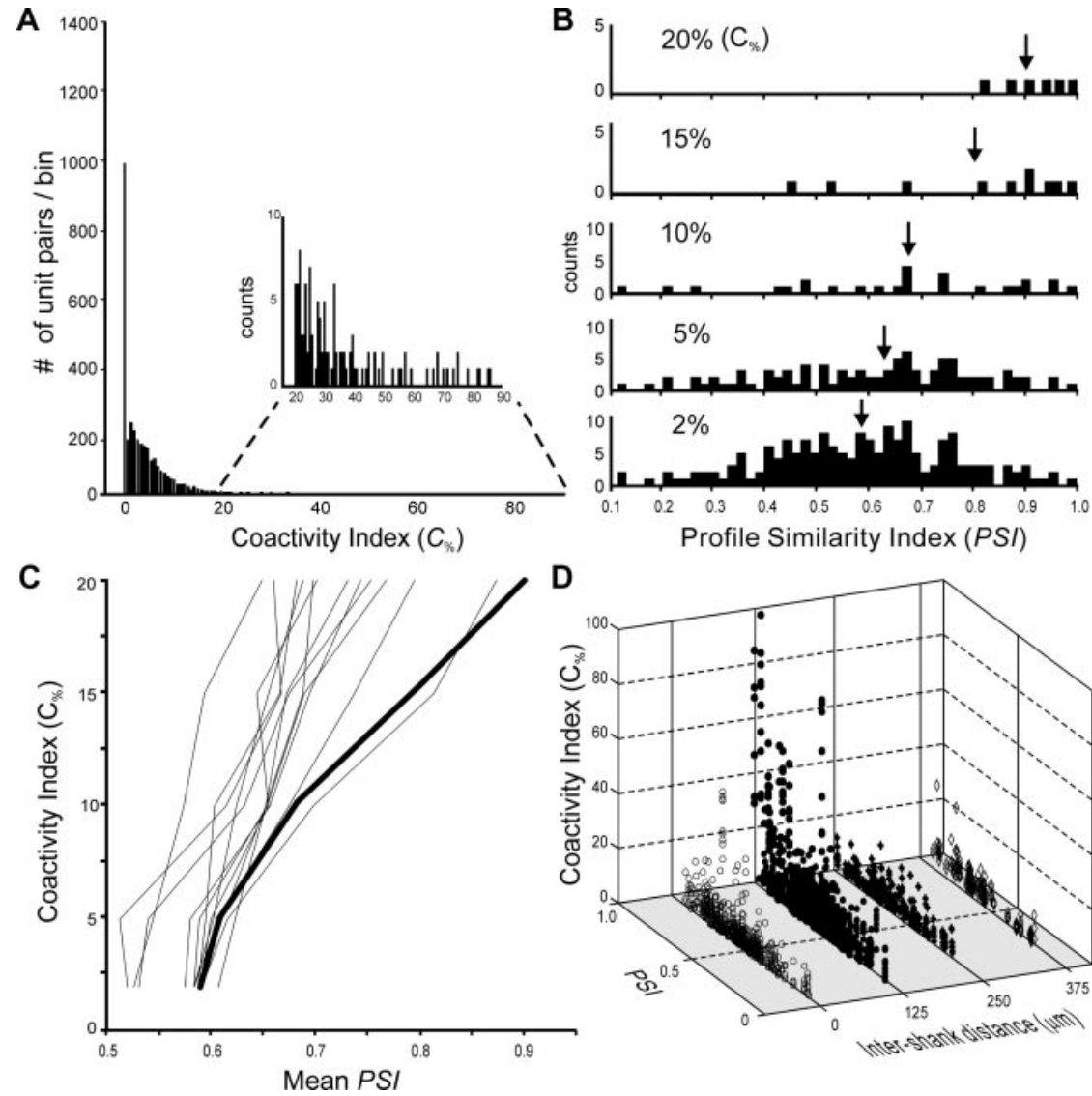

Figure 7. Relationship between profile similarity (PSI) and coactivity $\left(C_{\%}\right)$ across all units in all ensembles. Temporal correlations were quantified for each pair of units within each ensemble using Equation 3 to calculate $C_{\%}$, the shuffle-corrected coactivity index based on the percentage of coincident spikes of the total spike count (see Materials and Methods). $A$, Histogram showing the distribution of $C_{\%}$ values for the responses of all unit pairs across all odorants $(n=5464)$. The inset highlights the distribution of $C_{\%}$ values $\geq 20 \%$. $B$, Relationship between the PSI and $C_{\%}$ based on population data ( $n=535$ unit pairs) for all responses to lin. The series of histograms from the bottom to the top shows profile similarity distributions for progressively greater values of $C_{\%}$ ( $\geq 2$ to $\geq 20 \%$ ) calculated for all pairs of units. Arrows indicate mean values. C, Relationship between mean the PSI and $C_{\%}$ calculated for all odorants in the stimulus set. The linalool curve is shown as a thick line. D, PSI versus $C_{\%}$ relationships for each pair of unit responses to all odorants, separated according to intershank distance between recording sites. Odor-evoked transient synchrony occurred both within and across recording zones, with the greatest number of correlations occurring between units in adjacent zones (i.e., recording shanks separated by $125 \mu \mathrm{m}$ ).

Table 1. Relationship between interunit coactivity $\left(C_{\%}\right)$ and intershank distance

\begin{tabular}{lllll}
\hline \multicolumn{5}{l}{ Intershank separation $(\mu \mathrm{m})$} \\
\hline Preparation & 0 & 125 & 250 & 375 \\
\hline 1 & $4.85 \pm 0.82(\mathrm{a})$ & $\mathbf{1 0 . 0 6} \pm \mathbf{1 . 3 2}(\mathrm{b})$ & $2.05 \pm 0.82(\mathrm{a})$ & $3.05 \pm 0.72(\mathrm{a})$ \\
2 & $4.59 \pm 0.63(\mathrm{ab})$ & $6.43 \pm 0.41(\mathrm{~b})$ & $4.35 \pm 0.31(\mathrm{a})$ & $5.09 \pm 0.66(\mathrm{ab})$ \\
3 & $6.02 \pm 0.72(\mathrm{a})$ & $8.39 \pm 1.42(\mathrm{a})$ & $5.39 \pm 1.82(\mathrm{a})$ & $4.69 \pm 0.72(\mathrm{a})$ \\
4 & $6.66 \pm 0.92(\mathrm{a})$ & $8.54 \pm 0.47(\mathrm{~b})$ & $6.81 \pm 0.55(\mathrm{ab})$ & $6.78 \pm 1.01(\mathrm{ab})$ \\
5 & $4.69 \pm 0.69(\mathrm{a})$ & $\mathbf{8 . 6 0} \pm \mathbf{0 . 7 2}(\mathrm{b})$ & $4.61 \pm 0.53(\mathrm{a})$ & $3.93 \pm 0.56(\mathrm{a})$ \\
6 & $2.54 \pm 0.36(\mathrm{a})$ & $6.94 \pm 0.73(\mathrm{~b})$ & $5.92 \pm 0.80(\mathrm{ab})$ & $5.76 \pm 1.01(\mathrm{ab})$ \\
7 & $3.04 \pm 0.86(\mathrm{a})$ & $6.61 \pm 0.64(\mathrm{a})$ & $6.02 \pm 0.96(\mathrm{a})$ & $3.93 \pm 0.67(\mathrm{a})$ \\
8 & $3.62 \pm 0.40(\mathrm{a})$ & $\mathbf{1 1 . 6 2} \pm \mathbf{1 . 3 7}(\mathrm{b})$ & $4.32 \pm 0.35(\mathrm{a})$ & $5.72 \pm 0.73(\mathrm{a})$ \\
9 & $2.79 \pm 0.22(\mathrm{a})$ & $\mathbf{6 . 8 3} \pm \mathbf{0 . 5 0}(\mathrm{b})$ & $3.72 \pm 0.52(\mathrm{a})$ & $2.11 \pm 0.42(\mathrm{a})$ \\
10 & $6.74 \pm 1.13(\mathrm{a})$ & $7.96 \pm 1.03(\mathrm{a})$ & $5.66 \pm 1.01(\mathrm{a})$ & $3.46 \pm 0.83(\mathrm{a})$ \\
11 & $5.14 \pm 0.58(\mathrm{a})$ & $\mathbf{8 . 5 4} \pm \mathbf{0 . 7 3}(\mathrm{b})$ & $4.34 \pm 0.28(\mathrm{a})$ & $5.02 \pm 0.29(\mathrm{a})$ \\
12 & $6.10 \pm 1.27(\mathrm{a})$ & $\mathbf{1 0 . 0 9} \pm \mathbf{1 . 7 4}(\mathrm{b})$ & $6.53 \pm 0.93(\mathrm{a})$ & $4.18 \pm 1.16(\mathrm{a})$ \\
\hline
\end{tabular}

The average $C_{\%}$ values (mean $\pm \mathrm{SE}$ ) for each of the four shank separations for all odorants across all preparations (rows) are shown. Values within a row marked with the same letter are not significantly different (Kruskal-Wallis test followed by multiple comparisons; $p<0.05$ ). Values significantly greater than others in the same row are shown in bold. 
A

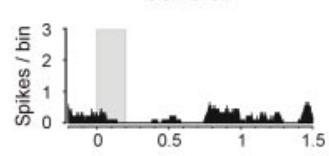

B
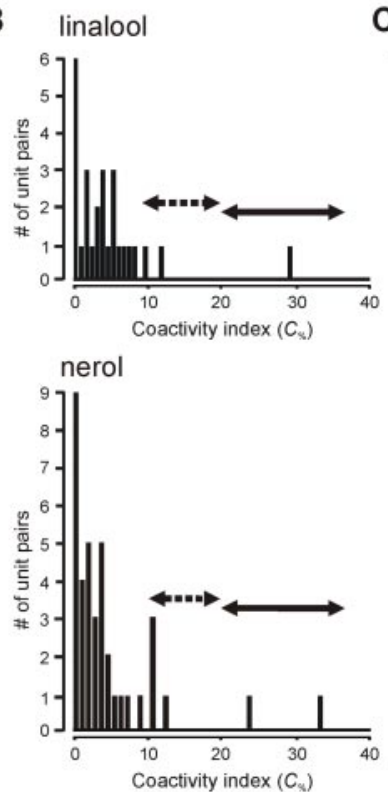$$
111-4
$$

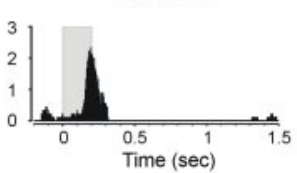

C linalool

$\mathrm{I}-1$
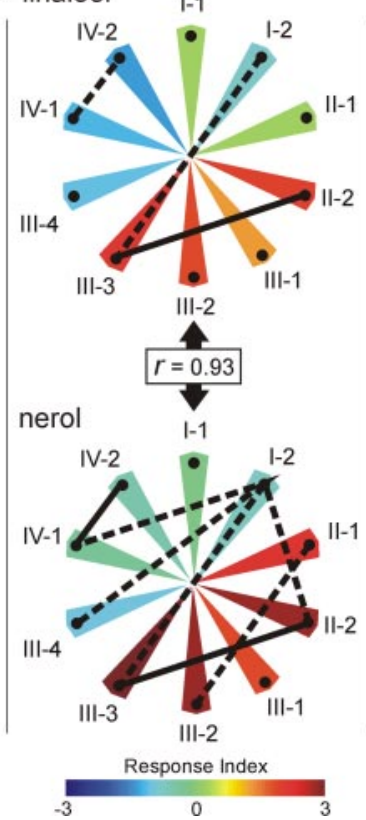

nerol

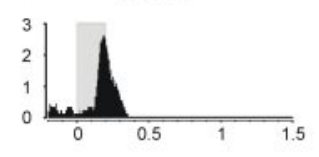

D linalool

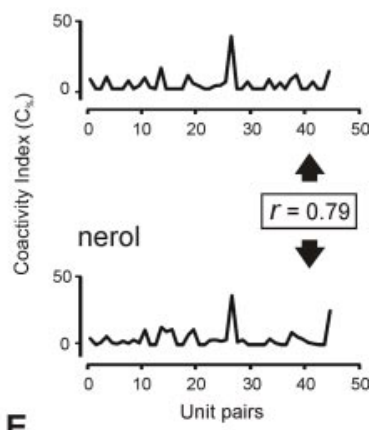

E

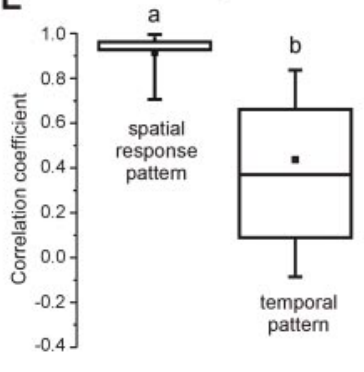

Figure 8. Integrating olfactory information in space and time. In this ensemble, the spatial patterns of population activity evoked by two similar stimulus compounds showed substantial overlap, but a distinct pattern of coincident firing embedded within the spatial pattern may further help distinguish one stimulus from another. A, In this unit (II-2), a 200 msec pulse (gray bar) of the monoterpenoid lin (middle), but not a control stimulus (left), evoked a brief burst of firing for each stimulus pulse. The monoterpenoid ner (right) evoked a response similar to the lin response. $B$, The index of coactivity $\left(C_{\%}\right)$ evoked by lin (top) and ner (bottom) was calculated for all possible pairs of units in this ensemble $(n=45)$. The frequency distributions for pairs with $C_{\%}$ values above (solid line) and below (dashed line) $20 \%$ are shown. Notice that the index of coactivity for the majority of pairs was $\leq 5 \%$. C, Comparing spatial response patterns with coactivity patterns. The spatial response pattern for the entire ensemble is represented as a circular matrix in which individual units are ordered in a clockwise direction starting from the 12:00 position (unit I-1). Each unit is represented as a wedge around the perimeter of the matrix, and its Rl is represented by the wedge color (see color scale). Also shown are the coactivity patterns (solid and dashed lines connecting unit pairs) that underlie the ensemble response to each stimulus. Note that many units showed no statistical increase in firing relative to the control response. Each connecting line represents the coactivity measure $\left(C_{\%}\right)$ between a specific pair of units. The spatial response patterns evoked by lin and ner are nearly identical $(r=0.93)$, but the coactivity patterns produced by these two odorants are distinctly different, with three pairs of units showing strong interactions in response to lin and a partially overlapping but distinct subset of seven unit pairs that showed correlations in response to ner. D, To quantify this difference, we converted the $C_{\%}$ values for lin and ner responses for all unit pairs $(n=45)$ into two vectors and then calculated the correlation coefficient between the vectors, revealing an $r$ value for the temporal correlation that was $15 \%$ lower than that for the spatial correlation. $E$, The same type of correlation analysis was performed across all animals $(n=12)$ to determine whether the observed relationship between spatial and temporal patterning may represent a general phenomenon. The first box chart compares the similarity between the response patterns evoked by lin and ner in terms of spatial patterning only. The high $r$ value indicates that these patterns are very similar and therefore difficult to distinguish. The second chart describes the similarity in temporal patterning after shuffle correction to account for coincident events that may be attributable only to elevated firing rates (see Materials and Methods). Each box chart shows the entire data range (error bars, 5th and 95 th percentiles; horizontal lines, 25 th, 50th, and 75 th percentiles; filled square, mean). The results indicate that although the spatial response patterns evoked by lin and ner are highly similar across all ensembles, the coactivity patterns representing these two odor stimuli provide a greater measure of discrimination (differences are statistically significant; $p<0.05$; Mann-Whitney $U$ test $; n=535$ unit pairs).

2004) and $\mathrm{CO}_{2}$ (Guerenstein et al., 2004) signals. Collectively, these data demonstrate that both $\mathrm{M} / \mathrm{T}$ and $\mathrm{PN}$ response patterns are, to a great extent, delimited by the temporal pattern of peripheral input to the glomerulus.

In one recent study examining pairs of $\mathrm{M} / \mathrm{T}$ cells separated by $300-500 \mu \mathrm{m}$ (the width of two to three glomeruli), 27\% of the pairs displayed odor-evoked synchronization, supporting the presence of local processing among glomeruli occupying the same region of the
OB (Kashiwadani et al., 1999). That study did not, however, involve systematic measurement of interelectrode distances. In the present study, we used a spatially distributed and precise recording array that allowed us to compare the response profiles and examine the temporal correlations between adjacent and nonadjacent cells simultaneously across the entire AL. Using this technique, we found direct evidence for local processing in Manduca, showing precise timing between nearby cells with a similar response profile, analogous to what has been reported in the mammalian OB (Mori et al., 1992). Of the 125 unit pairs with a $C_{\%}$ value $\geq 20$ (Fig. $7 A$ ), $95 \%$ were localized to the same or neighboring zones of glomerular neuropil. These local interactions have been predicted from both empirical and modeling studies in Manduca (Christensen et al., 2001; Reisenman et al., 2004; Guerenstein et al., 2004), and they are furthermore consistent with paired recording results from other vertebrate and invertebrate studies (Schoppa and Westbrook, 2001; Lei et al., 2002). Additional analysis of the temporal relationships between units revealed for each stimulus a distinct pattern of unit coactivity across the ensemble, and each pattern could be clearly distinguished by which units were correlated as well as by the strength of these correlations (Fig. 8E) (supplemental Fig. 1, available at www.jneurosci.org as supplemental material). The strongest synchrony occurred between neurons that showed statistically significant responses based on their PSTHs, but weaker correlations also occurred between nonresponsive neurons, suggesting that even neurons that are not statistically responsive may nevertheless participate in the coding ensemble through temporal coordination of their firing (Fig. 8C). Multichannel ensemble recording thus revealed odordependent "subthreshold" temporal interactions that may be below the resolution of popular activity-labeling methods. Collectively, our findings using neural-ensemble recording support the hypothesis that the spatial extent of stimulus-evoked activity in the olfactory system is defined primarily by the particular subset of glomeruli activated by a given stimulus. Moreover, they provide new evidence that local processing within and between neighboring glomeruli may further enhance discrimination, especially among chemically similar odorants.

\section{References}

Adrian ED (1942) Olfactory reactions in the brain of the hedgehog. J Physiol (Lond) 100:459-473.

Aertsen AMHJ, Gerstein GL, Habib M, Palm G (1989) Dynamics of neuronal firing correlation: modulation of "effective connectivity." J Neurophysiol 61:900-917.

Aungst JL, Heyward PM, Puche AC, Karnup SV, Hayar A, Szabo G, Shipley 
MT (2003) Centre-surround inhibition among olfactory bulb glomeruli. Nature 426:623-629.

Brown EN, Kass RE, Mitra PP (2004) Multiple neural spike train data analysis: state-of-the-art and future challenges. Nat Neurosci 7:456-461.

Buonviso N, Chaput MA (1990) Response similarity to odors in olfactory bulb output cells presumed to be connected to the same glomerulus: electrophysiological study using simultaneous single unit recordings. J Neurophysiol 63:447-454.

Cang J, Isaacson JS (2003) In vivo whole-cell recording of odor-evoked synaptic transmission in the rat olfactory bulb. J Neurosci 23:4108-4116.

Carlson GC, Shipley MT, Keller A (2000) Long-lasting depolarizations in mitral cells of the rat olfactory bulb. J Neurosci 20:2011-2021.

Christensen TA, Hildebrand JG (2002) Pheromonal and host-odor processing in the insect antennal lobe: how different? Curr Opin Neurobiol 12:393-399.

Christensen TA, White J (2000) Representation of olfactory information in the brain. In: The neurobiology of taste and smell (Finger TE, Silver WL, Restrepo D, eds), pp 201-232. New York: Wiley.

Christensen TA, Waldrop BR, Harrow ID, Hildebrand JG (1993) Local interneurons and information processing in the olfactory glomeruli of the moth Manduca sexta. J Comp Physiol [A] 173:385-399.

Christensen TA, Waldrop BR, Hildebrand JG (1998) Multitasking in the olfactory system: context-dependent responses to odors reveal dual GABA-regulated coding mechanisms in single olfactory projection neurons. J Neurosci 18:5999-6008.

Christensen TA, Pawlowski VM, Lei H, Hildebrand JG (2000) Multi-unit recordings reveal context-dependent modulation of synchrony in odorspecific ensembles. Nat Neurosci 3:927-931.

Christensen TA, D’Alessandro G, Lega J, Hildebrand JG (2001) Morphometric modeling of olfactory circuits in the insect antennal lobe: I. Simulation of spiking local interneurons. Biosystems 61:143-153.

Christensen TA, Lei H, Hildebrand JG (2003) Coordination of central odor representations through transient, non-oscillatory synchronization of glomerular output neurons. Proc Natl Acad Sci USA 100:11076-11081.

Daly KC, Durtschi ML, Smith BH (2001) Olfactory-based discrimination learning in the moth, Manduca sexta. J Insect Physiol 47:375-384.

Daly KC, Wright GA, Smith BH (2004a) Molecular features of odorants systematically influence slow temporal responses across clusters of coordinated antennal lobe units in the moth Manduca sexta. J Neurophysiol 92:236-254.

Daly KC, Christensen TA, Lei H, Smith BH, Hildebrand JG (2004b) Learning modulates the ensemble representations for odors in primary olfactory networks. Proc Natl Acad Sci USA 101:10476-10481.

Fletcher ML, Wilson D (2003) Olfactory bulb mitral-tufted cell plasticity: odorant-specific tuning reflects previous odorant exposure. J Neurosci 23:6946-6955.

Fraser AM, Mechaber W, Hildebrand JG (2003) Electroantennographic and behavioral responses of the sphinx moth Manduca sexta to host plant headspace volatiles. J Chem Ecol 29:1813-1833.

Freeman W (1992) Nonlinear dynamics in olfactory information processing. In: Olfaction, a model system for computational neuroscience (Davis J, Eichenbaum H, eds), pp 225-249. Cambridge, MA: MIT.

Fuss SH, Korsching SI (2001) Odorant feature detection: activity mapping of structure response relationships in the zebrafish olfactory bulb. J Neurosci 21:8396-8407.

Galizia GC, Menzel R (2001) The role of glomeruli in the neural representation of odours: results from optical recording studies. J Insect Physiol 47:115-130.

Gao Q, Yuan B, Chess A (2000) Convergent projections of Drosophila olfactory neurons to specific glomeruli in the antennal lobe. Nat Neurosci 3:780-785.

Gelperin A, Tank DW (1990) Odor-modulated collective network oscillations of olfactory interneurons in a terrestrial mollusc. Nature 345:437-440.

Guerenstein P, Christensen TA, Hildebrand JG (2004) Sensory processing of ambient- $\mathrm{CO}_{2}$ information in the brain of the moth Manduca sexta. J Comp Physiol [A] 190:707-725.

Hansson BS, Carlsson MA, Kalinovà B (2003) Olfactory activation patterns in the antennallobe of the sphinx moth, Manduca sexta. J Comp Physiol [A] 189:301-308.

Inaki K, Takahashi YK, Nagayama S, Mori K (2002) Molecular-feature domains with posterodorsal-anteroventral polarity in the symmetrical sen- sory maps of the mouse olfactory bulb: mapping of odourant-induced Zif268 expression. Eur J Neurosci 15:1563-1574.

Johnson BA, Leon M (2000a) Modular representations of odorants in the glomerular layer of the rat olfactory bulb and the effects of stimulus concentrations. J Comp Neurol 422:496-509.

Johnson BA, Leon M (2000b) Odorant molecular length: one aspect of the olfactory code. J Comp Neurol 426:330-338.

Johnson BA, Ho S, Xu Z, Yihan J, Yip S, Hingco E, Leon M (2002) Functional mapping of the rat olfactory bulb using diverse odorants reveals modular responses to functional groups and hydrocarbon structural features. J Comp Neurol 449:180-194.

Kaissling KE, Hildebrand JG, Tumlinson JH (1989) Pheromone receptor cells in the male moth Manduca sexta. Arch Insect Biochem Physiol 10:273-279.

Kashiwadani H, Sasaki YF, Uchida N, Mori K (1999) Synchronized oscillatory discharges of mitral/tufted cells with different molecular receptive ranges in the rabbit olfactory bulb. J Neurophysiol 82:1786-1792.

Kass RE, Ventura V, Cai C (2003) Statistical smoothing of neuronal data. Network: Comput Neural Syst 14:5-15.

Keller A, Vosshall LB (2003) Decoding olfaction in Drosophila. Curr Opin Neurobiol 13:103-110.

Korsching S (2002) Olfactory maps and odor images. Curr Opin Neurobiol 12:387-392.

Laurent G, Stopfer M, Friedrich RW, Rabinovich MI, Volkovskii A, Abarbanel HD (2001) Odor encoding as an active, dynamical process: experiments, computation, and theory. Annu Rev Neurosci 24:263-297.

Lehmkuhle MJ, Normann RA, Maynard EM (2003) High-resolution analysis of the spatio-temporal activity patterns in rat olfactory bulb evoked by enantiomer odors. Chem Senses 28:499-508.

Lei H, Christensen TA, Hildebrand JG (2002) Local inhibition modulates odor-evoked synchronization of glomerulus-specific output neurons. Nat Neurosci 5:557-565.

Linster C, Johnson BA, Yue E, Morse A, Xu Z, Hingco EE, Choi Y, Choi M, Messiha A, Leon M (2001) Perceptual correlates of neural representations evoked by odorant enantiomers. J Neurosci 21:9837-9843.

Margrie TW, Schaefer AT (2003) Theta oscillation coupled spike latencies yield computational vigour in a mammalian sensory system. J Physiol (Lond) 546:363-374.

Marin EC, Jefferis GSXE, Komiyama T, Zhu H, Luo L (2002) Representation of the glomerular olfactory map in the Drosophila brain. Cell 109:243-255.

Meijerink J, Carlsson MA, Hansson BS (2003) Spatial representation of odorant structure in the moth antennal lobe: a study of structureresponse relationships at low doses. J Comp Neurol 467:11-21.

Mori K, Mataga N, Imamura K (1992) Differential specificities of single mitral cells in rabbit olfactory bulb for a homologous series of fatty acid odor molecules. J Neurophysiol 67:786-789.

Müller D, Abel R, Brandt R, Zockler M, Menzel R (2002) Differential parallel processing of olfactory information in the honeybee, Apis mellifera $\mathrm{L}$. J Comp Physiol [A] 188:359-370.

Ng M, Roorda RD, Lima SQ, Zemelman BV, Morcillo P, Miesenbock G (2002) Transmission of olfactory information between three populations of neurons in the antennal lobe of the fly. Neuron 36:463-474.

Nikonov AA, Caprio J (2001) Electrophysiological evidence for a chemotopy of biologically relevant odors in the olfactory bulb of the channel catfish. J Neurophysiol 86:1869-1876.

Reisenman CE, Christensen TA, Francke W, Hildebrand JG (2004) Enantioselectivity of projection neurons innervating identified olfactory glomeruli. J Neurosci 24:2602-2611.

Rospars JP, Hildebrand JG (2000) Sexually dimorphic and isomorphic glomeruli in the antennal lobes of the sphinx moth Manduca sexta. Chem Senses 25:119-129.

Rubin BD, Katz LC (1999) Optical imaging of odorant representations in the mammalian olfactory bulb. Neuron 23:499-511.

Sachse S, Galizia CG (2002) Role of inhibition for temporal and spatial odor representation in olfactory output neurons: a calcium imaging study. J Neurophysiol 87:1106-1117.

Sachse S, Galizia CG (2003) The coding of odour-intensity in the honeybee antennal lobe: local computation optimizes odour representation. Eur J Neurosci 18:2119-2132.

Schoppa N, Urban NN (2003) Dendritic processing within olfactory bulb circuits. Trends Neurosci 26:501-506. 
Schoppa NE, Westbrook GL (2001) Glomerulus-specific synchronization of mitral cells in the olfactory bulb. Neuron 31:639-651.

Shields VDC, Hildebrand JG (2001) Responses of a population of antennal olfactory receptor cells in the female moth Manduca sexta to plantassociated volatile organic compounds. J Comp Physiol [A] 186:1135-1151.

Sobel EC, Tank DW (1993) Timing of odor stimulation does not alter patterning of olfactory bulb unit activity in freely breathing rats. J Neurophysiol 69:1331-1337.

Spors H, Grinvald A (2002) Spatio-temporal dynamics of odor representations in the mamalian olfactory bulb. Neuron 34:301-315.

Stopfer M, Bhagavan S, Smith B, Laurent G (1997) Impaired odour discrimination on desynchronization of odour-encoding neural assemblies. Nature 390:70-74.

Stopfer M, Jayraman V, Laurent G (2003) Intensity versus identity coding in an olfactory system. Neuron 39:991-1004.

Theunissen FE (2003) From synchrony to sparseness. Trends Neurosci 26:61-64.

Tolbert LP, Hildebrand JG (1981) Organization and synaptic ultrastructure of glomeruli in the antennal lobes of the moth Manduca sexta: a study using thin sections and freeze-fracture. Proc R Soc Lond B Biol Sci 213:279-301.

Tsuboi A, Yoshihara S, Yamazaki N, Kasai H, Asai-Tsuboi H, Komatsu M, Serizawa S, Ishii T, Matsuda Y, Nagawa F, Sakano H (1999) Olfactory neurons expressing closely linked and homologous odorant receptor genes tend to project their axons to neighboring glomeruli on the olfactory bulb. J Neurosci 19:8409-8418.

Uchida N, Takahashi YK, Tanifuji M, Mori K (2000) Odor maps in the mammalian olfactory bulb: domain organization and odorant structural features. Nat Neurosci 3:1035-1043.

Ushiba J, Tomita Y, Masakado Y, Komune Y (2002) A cumulative sum test for a peri-stimulus time histogram using the Monte Carlo method. J Neurosci Methods 118:207-214.

Vosshall LB, Wong AM, Axel R (2000) An olfactory sensory map in the fly brain. Cell 102:147-159.

Wachowiak M, Cohen LB (2001) Representation of odorants by receptor neuron input to the mouse olfactory bulb. Neuron 32:723-735.

Wehr M, Laurent G (1996) Odour encoding by temporal sequences of firing in oscillating neural assemblies. Nature 384:162-166.

Wilson MA, McNaughton BL (1994) Reactivation of hippocampal ensemble memories during sleep. Science 265:676-679.

Wong AM, Wang JW, Axel R (2002) Spatial representation of the glomerular map in the Drosophila protocerebrum. Cell 109:229-241.

Xu F, Liu N, Kida I, Rothman DL, Hyder F, Shepherd GM (2003) Odor maps of aldehydes and esters revealed by functional MRI in the glomerular layer of the mouse olfactory bulb. Proc Natl Acad Sci USA 100:11029-11034.

Yokoi M, Mori K, Nakanishi S (1995) Refinement of odor molecule tuning by dendrodendritic synaptic inhibition in the olfactory bulb. Proc Natl Acad Sci USA 92:3371-3375. 